\title{
Factores biológicos en pacientes con Trastorno de Estrés Postraumático (PTSD) y Alcoholismo
}

\author{
Vlatko Thaller \\ Srdan Marušić \\ Križo Katinić \\ Danijel Buljan \\ Vesna Golik-Gruber \\ Jelena Potkonjak
}

Departamento Universitario de Psiquiatría KB "Sestre Milosrdnice", Referal Centre for Alcoholism Ministry of Health, 10000 Zagreb, Vinogradska c. 29 CROACIA

\footnotetext{
RESUMEN - Los acontecimientos traumáticos que sobrepasan la experiencia humana normal, tales como las situaciones que ponen en peligro la vida o la integridad física o la exposición de personas próximas a un peligro similar, pueden producir determinados problemas psicológicos en el individuo, que se describen en el marco de un trastorno denominado Trastorno de Estrés Postraumático. La exposición a numerosos acontecimientos e influencias estresantes produce, como resultado de numerosos factores, el que el organismo desencadene una serie de mecanismos de defensa, en los que juega un papel fundamental el Sistema Nervioso Central, pero también el sistema suprarrenal y el cardiorespiratorio. Cuando la homeostasis interna está en peligro, se activa el sistema endocrino y su respuesta al peligro depende de la intensidad del agente causante del estrés, de la duración de la exposición y de la capacidad del sistema para compensar el estrés. La exposición al estrés y el efecto directo del agente causante de estrés desencadena además otro mecanismo de defensa, cual es el aumento del consumo de alcohol a modo de automedicación y bajo la influencia directa del agente causante de estrés. Tanto el PTSD como el alcoholismo conducen de forma gradual a la afectación del sistema neuroendocrino. En nuestro estudio hemos incluido dos grupos de pacientes, concretamente el grupo de pacientes que padecen de PTSD y de alcoholismo y el grupo de pacientes que padecen únicamente de PTSD. Hemos llevado a cabo la evaluación de los niveles de cortisol en suero y en la orina de 24 horas, el test de supresión de la dexametasona y los niveles en suero de ACTH, TSH, $\mathrm{T} 3, \mathrm{~T} 4$, testosterona, prolactina y hormona del crecimiento.

Los resultados obtenidos llevan a considerar la presencia de alteraciones en los ejes neuroendocrinos en los dos grupos de esta investigación, alteraciones que se presentan más marcadas en el grupo de sujetos que presentan comorbilidad de PTSD y alcoholismo.
} 


\section{Introducción}

Los acontecimientos traumáticos que van más allá de la experiencia humana normal, tales como son las situaciones en las que la vida se ve amenazada, las de peligro para la integridad física de las personas pueden afectar al individuo hasta el punto de causar determinados problemas psicológicos, descritos en el cuadro de la enfermedad denominada Trastorno de Estrés Postraumático (PTSD) (DSMIV American Psychiatric Association 1994).

Los síntomas presentes son causados por la influencia combinada de factores biológicos y psicológicos. El cuadro clínico se caracteriza por la presencia de ansiedad, temor, escenas retrospectivas (flashbacks) de acontecimientos traumáticos pasados, depresión, disminución de la capacidad de concentración e irritabilidad.

La exposición cotidiana a una serie de acontecimientos e influencias estresantes produce, como resultado de numerosos factores, el desarrollo de mecanismos de defensa por parte del organismo.

El mantenimiento del equilibrio del estado interno con una relativa estabilidad se denomina homeostasis y es importante para la supervivencia. Este frágil equilibrio de las funciones bioquímicas y psicológicas del organismo se ve desafiado constantemente por la extensa gama de factores causantes de estrés.

Como respuesta al estrés, el organismo activa una serie de cambios fisiológicos y de cambios en la conducta, que en realidad suponen un proceso de adaptación con el objetivo de mantener la homeostasis y de mejorar la capacidad de enfrentarse con el estrés.

La respuesta del organismo ante el estrés es una red integrada de extraordinaria complejidad en la que intervienen el Sistema
Nervioso Central (CNS), el sistema suprarrenal y los sistemas circulatorio y respiratorio. Cuando la homeostasis interna del organismo se encuentra en peligro, el hipotálamo inicia la secreción del factor de liberación adrenocorticotrópica (hormona CRF), que coordina la respuesta del organismo ante el estrés por medio de reacciones fisiológicas y de conducta complejas e interrelacionadas. La hormona CRF activa la hipófisis que libera la hormona adrenocorticotrópica (ACTH), que inicia la secreción de hormonas glucocorticoides procedentes de las glándulas suprarrenales (cortisol). Dichas hormonas juegan un papel clave en la respuesta del organismo frente al estrés y en su cese. (Tsigos \& Chrousos 1995, De Kloet et al. 1987, Oitzl et al. 1997).

Estas hormonas afectan a las funciones de los músculos lisos (o involuntarios), a la regulación del metabolismo de las grasas, a las funciones del tracto gastrointestinal, a los riñones y a otros muchos órganos y funciones del organismo tales como la regulación de la temperatura, el apetito, la estimulación, el insomnio, la atención y el estado de ánimo. La adaptación física al estrés hace posible que el organismo redirija el aporte de oxígeno, es decir, las sustancias nutritivas, hacia el lugar del organismo que padece el estrés, precisamente en donde son más necesarias (Tsigous \& Rousos 1995). De este modo el papel del eje formado por el hipotálamo, la hipófisis y las glándulas suprarrenales (HHA) es importante en el desarrollo de los síntomas presentes en el Trastorno de Estrés Postraumático. La exposición al estrés se ve afectada no sólo por la función adrenocorticotrópica, sino que existen otras hormonas que juegan también un importante papel, como son las hormonas tiroideas, las hormonas gonadotrópicas, la prolactina y los péptidos opioides (Khansari et al. 1990). Asimismo, numero- 
sos estudios realizados en los últimos veinte años, muestran como existe una conexión entre el estrés psicológico, las enfermedades psicológicas y el estado y funcionamiento del sistema inmunológico (Bonneus et al. 1990, Owens et al. 1996).

Estudios sobre la importancia del eje HHA en pacientes con Trastorno de Estrés Postraumático (PTSD) muestran que la respuesta ACTH al CRF disminuye, aunque también se han observado incrementos de CRF (Gold et al. 1986, Southwick et al. 1992).

La mayoría de los pacientes que sufren PTSD muestran un incremento en la actividad de CRF en el hipotálamo o una disminución en la respuesta ACTH al CRF (Yehuda 1996). Puesto que se sabe que el CRF inyectado en los ventrículos de los animales de laboratorio produce un efecto similar al comportamiento de pacientes con PTSD, podríamos suponer que el mismo podría ser responsable de algunos de los síntomas de pacientes que sufren PTSD. Deberíamos subrayar además el hecho de que, como se evidencia por numerosos estudios relacionados con el sistema neuroendocrino en pacientes con PTSD, parece haber una reacción compensatoria de CNS, a través del eje HHA, en la que el aumento de la secreción de cortisol durante un periodo de tiempo prolongado puede dar lugar a la disfunción de la respuesta de cortisol al hipotálamo, originando la hipersecreción de cortisol, con el resultado final de la alteración del eje HHA (Yehuda et al. 1991, Constanti et al. 1998, Friedman 1991, Vrkljan et al. 1997). Con frecuencia, en pacientes que padecen de un PTSD, podemos encontrar alcoholismo, es decir, la comorbilidad de alcoholismo y PTSD, complicación considerable que origina una mayor gravedad en los pacientes y complica el adecuado proceso de tratamiento.

Varios estudios muestran como la incidencia del abuso de alcohol y el alcoholismo está en correlación con diversos factores conectados con el estrés, concretamente con los factores genéticos, con la actitud del individuo hacia el alcoholismo con anterioridad a los acontecimientos traumáticos, con las expectativas subjetivas sobre el efecto del alcohol sobre el estrés, con la intensidad y el tipo de agente causante del estrés y con la disponibilidad del apoyo social general encaminado a aliviar el efecto del estrés. Además, algunas investigaciones han mostrado como un elevado grado de estrés puede ser causa de un aumento de la ingestión de alcohol si no se dispone de una solución más adecuada, si se puede tener acceso al alcohol con facilidad y si la persona considera que el alcohol puede ayudarle a reducir el efecto del estrés (Jenison et al. 1992, Sadawa \& Pak 1993, Davidson et al. 1990).

Según algunos autores, dosis reducidas de alcohol pueden reducir la intensidad de respuesta al estrés, pero también hay individuos en los que, una determinada cantidad de alcohol, ocasiona de forma más probable un aumento que una reducción de la respuesta del organismo al estrés, estimulando la actividad del eje hipófisis-hipotálamo. El consumo de alcohol tiene como consecuencia un incremento del nivel sérico de corticosteroides. $\mathrm{La}$ coexistencia de daños hepáticos, malnutrición, depresión y otros agentes causantes de estrés, así como la interacción permanente de la intoxicación y desintoxicación, afectan también a los resultados de la interacción entre el alcohol y el eje HHA. Estudios posteriores revelaron el incremento inmediato de los niveles séricos de cortisol, dando como resultado un tipo de pseudo-síndrome de Cushing, confirmando de forma clara la tesis de que el HHA se encuentra dañado en los alcohólicos (Wand \& Dobbs 1991). También se han detectado en alcohólicos alteraciones en la producción de la hormona TRH, que afectando a la hipófisis se induce la secreción 
de la hormona estimulante del tiroides (TSH), que en consecuencia estimula al tiroides para que libere las hormonas conocidas como T3 y T4 (Loosen 1988). El hecho de que la ingestión de alcohol reduce los niveles séricos de la hormona T4 parece indicar que el eje HHA presenta alteraciones en los alcohólicos. De esta forma, la ingestión de alcohol afecta al eje HHA de forma similar a como lo hacen los factores causantes de estrés, teniendo como resultado la alteración de su función, si bien las alteraciones de otros ejes neuroendocrinos que se producen en el curso del alcoholismo y del PTSD tampoco deberían omitirse, esto es, las alteraciones en el eje hipotálamo-hipófisis-tiroides (HHT) y el eje hipotálamo-hipófisis-gonadal (HHGn) (Emanuel \& Emanuel 1997). Los nuevos datos acerca de las alteraciones que se presentan como reacción al estrés intenso a nivel del eje HHA, nos obligan a considerar que las reacciones de las personas ante el estrés son diversas y varían entre individuos, sin actuar nunca de acuerdo con una pauta única, mostrando que el incremento de la actividad de CRF guarda relación con el incremento de la secreción de cortisol. La intensidad y la orientación de la respuesta hipófisis-suprarrenal al estrés podrían depender de la interacción de algunos factores, incluyendo la naturaleza del estrés, la exposición previa al estrés, los factores sociales y la predisposición genética. Los efectos de estos factores pueden alterar en gran medida también la respuesta del eje HHA al estrés en un mismo individuo (Tsigos \& Chrousos 1995).

\section{Objetivo y métodos}

Teniendo en cuenta los estudios previos en torno a las alteraciones del sistema neuroendocrino en pacientes que padecen
PTSD y alcoholismo, hemos decidido llevar a cabo un estudio de investigación de la función del sistema neuroendocrino, para confirmar los hallazgos de que el alcoholismo y el PTSD pueden producir daños en el sistema neuroendocrino y en consecuencia la perturbación de la producción hormonal, afecta a la regulación de las funciones mentales y de ciertos tipos de conducta. En nuestro estudio, hemos incluido un grupo de pacientes que padecen Trastorno de Estrés Postraumático (PTSD) $(\mathrm{N}=34)$ y otro grupo, integrado por pacientes que padecen tanto PTSD como alcoholismo $(\mathrm{N}=31)$.

Hemos llevado a cabo los procedimientos de examen y diagnóstico clínicos para los dos grupos de pacientes objeto de estudio, según los criterios DSM-IV relativos a la dependencia del alcohol y al PTSD y tambien de acuerdo con nuestros propios cuestionarios adicionales con relación a PTSD y al alcoholismo. Todos los pacientes eran veteranos de guerra croatas. Se han medido los niveles de cortisol en la muestra de orina recogida durante 24 horas (los valores normales para nuestros laboratorios oscilan entre $250-750 \mathrm{nmol} / \mathrm{dV}$ ). También realizamos la medición de los niveles séricos de cortisol a las 8:00 horas y a las 16:00 horas, teniendo en cuenta el hecho de que el nivel de cortisol es más elevado por la mañana y más bajo por la noche (en torno al $30 \%$ más bajo comparado con los niveles de la mañana, a ritmo circadiano* normal). Los niveles séricos de cortisol normales para nuestros laboratorios oscilan entre 130-800 nmol/1. Seguidamente medimos los niveles séricos de hormona T3 (los valores normales van de 1,1 a 2,8 nmol/1), la hormona T4 (valores normales entre $50-165 \mathrm{nmol} / \mathrm{l}$ ) y TSH (valores normales de 0,1 a 4,0 n/J/l). Además de estas hormonas tiroideas, medimos también los niveles séricos de prolactina (valores normales en hombres oscilando entre $5 \mathrm{y}$ 
25 ng/l). A continuación medimos los niveles séricos de testosterona (valores normales para hombres adultos entre 10,5 y 49,0), ACTH (valores normales oscilando entre 2,0 y 13,3 pmol/l) y hormona del crecimiento (valores normales entre 0 y $5 \mathrm{ng} / \mathrm{l}$ ).

Hemos realizado también el test de la dexametasona en ambos grupos de pacientes, es decir, administramos a los pacientes, por vía oral, 2 píldoras de $0,5 \mathrm{mg}$ de dexametasona inmediatamente antes de acostarse (a las 23:00 h aproximadamente) y las muestras de extracción de sangre se tomaron a las 8:00 h y las 16:00 h del día siguiente. Los valores normales de cortisol en la prueba de supresión de dexametasona son inferiores a $80 \mathrm{nmol} / \mathrm{l}$.

Los resultados se han mostrado utilizando gráficos de caja y extensión. La importancia estadística de las diferencias se ha establecido por la comparación de los valores medios en los dos grupos de sujetos, utilizando el test-T de Student para muestras independientes, con probabilidad estadística establecida en $\mathrm{p}<0,05$. Hemos denominado a los grupos PTSP-A (pacientes con síntomas del cuadro de PTSD) y ALK-B (pacientes en los que concurren alcoholismo y PTSD).

\section{Resultados}

Las figuras 1 y 2 muestran los niveles séricos de cortisol a las 8:00 horas y a las 16:00 horas en ambos grupos. Nuestros resultados mostraron que los niveles en suero de cortisol son similares a las 8:00 horas, mostrando en ambos casos un ligero aumento en los niveles, pero se da una diferencia significativa a las 16:00 horas, momento en que el grupo ALK-B mostró niveles de cortisol en suero significativamente más elevados. Hemos observado diferencias circadianas más pronunciadas en ambos grupos de pacientes (una diferencia mayor entre los niveles de la mañana y de la tarde), particularmente en el grupo ALK-B. La figura 3 muestra los niveles de cortisol en orina de 24 horas. Los resultados muestran incrementos en los niveles de cortisol de 24 horas en ambos grupos, lo que hace suponer una disminución de sensibilidad a la respuesta negativa.

La figura 6 muestra los niveles de ACTH en suero, que eran bastante similares en los dos grupos de individuos objeto de estudio, ambos en la parte superior de la gama de valores normales. Las figuras 7, 8 y 9 muestran los nive-

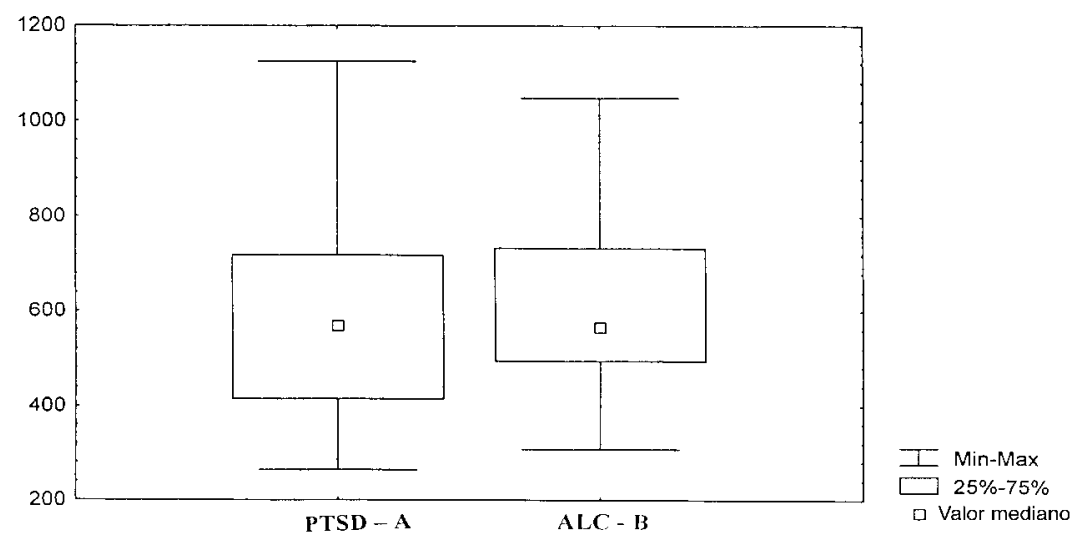

Figura 1. Niveles en suero de Cortisol medidos a las 8:00 h en el grupo de pacientes con PTSD (A) y en el grupo de pacientes con ambos diagnósticos, alcoholismo y PTSD (B). 

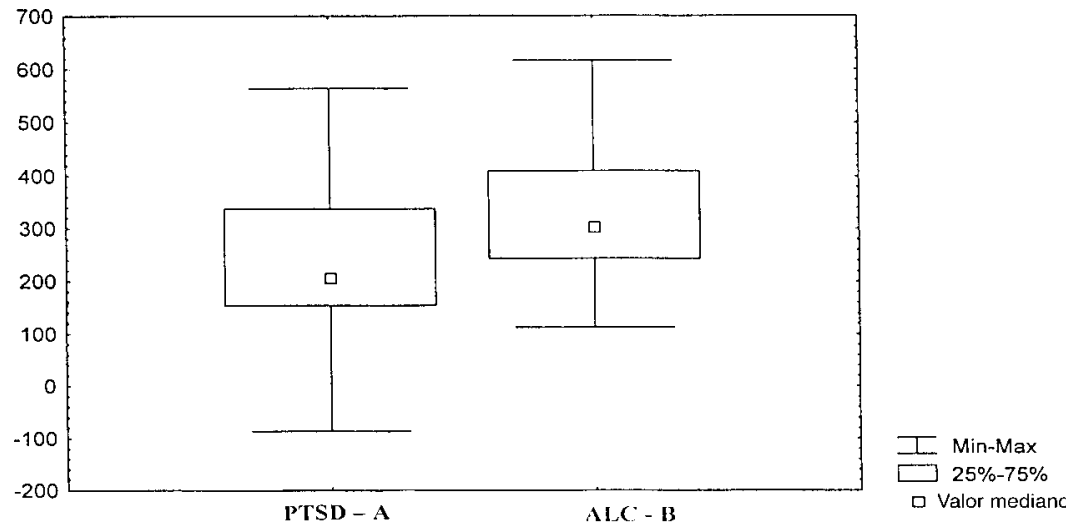

Figura 2. Niveles en suero de Cortisol medidos a las 16:00 h en el grupo de pacientes con PTSD (A) y en el grupo de pacientes con ambos diagnósticos, alcoholismo y PTSD (B).

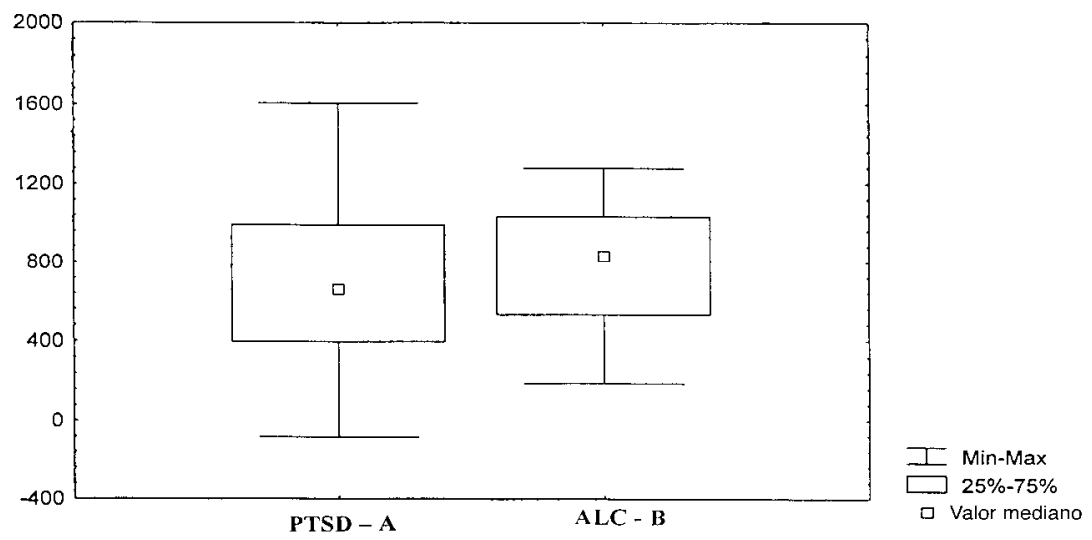

Figura 3. Niveles en orina de Cortisol en el grupo de pacientes con PTSD (A) y en el grupo de pacientes con ambos diagnósticos, alcoholismo y PTSD (B).

les en suero de T3, T4 y TSH. Los niveles de TSH resultaron ser significativamente más altos en el grupo ALK-B. Los niveles en suero de T3 y T4 también eran significativamente más elevados en el grupo ALK-B. La figura 10 muestra los niveles en suero de testosterona en ambos grupos. Los valores medios son similares, en ambos casos en la zona superior de los valores normales. La figura 11 muestra los niveles en suero de la hormona de crecimiento.
Los resultados han mostrado el aumento en suero de la hormona de crecimiento en el grupo ALK-B. La diferencia es estadísticamente representativa $(\mathrm{p}<0,05)$.

La figura 12 muestra los niveles séricos de prolactina en ambos grupos de los individuos objeto de estudio. Los resultados demuestran que los niveles de prolactina son significativamente más bajos en el grupo ALK-B en comparación con el grupo PTSP-A. 


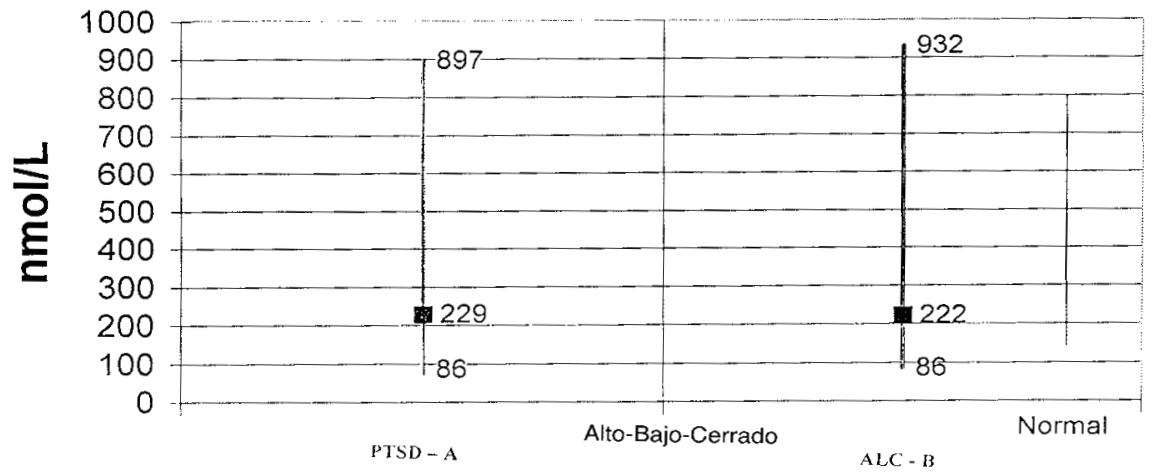

Figura 4. Niveles séricos de Cortisol en el test de Dexametasona en el grupo de pacientes con PTSD (A) y en el grupo de pacientes con ambos diagnósticos de alcoholismo y PTSD (B) medidos a las 8:00 h.

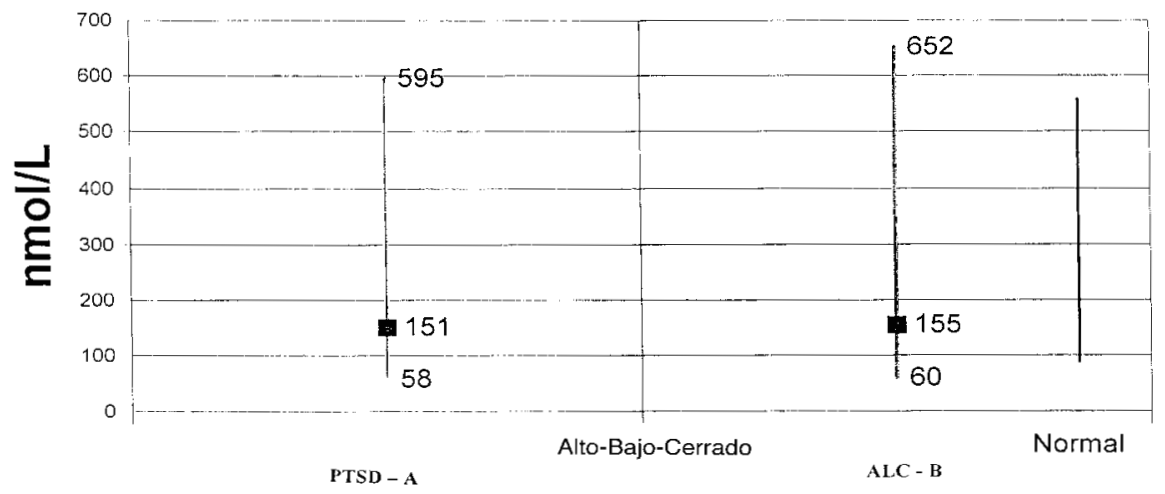

Figura 5. Niveles de Cortisol en suero el test de Dexametasona en el grupo de pacientes con PTSD (A) y en el grupo de pacientes con ambos diagnósticos, alcoholismo y PTSD (B) medidos a las 16:00 h.

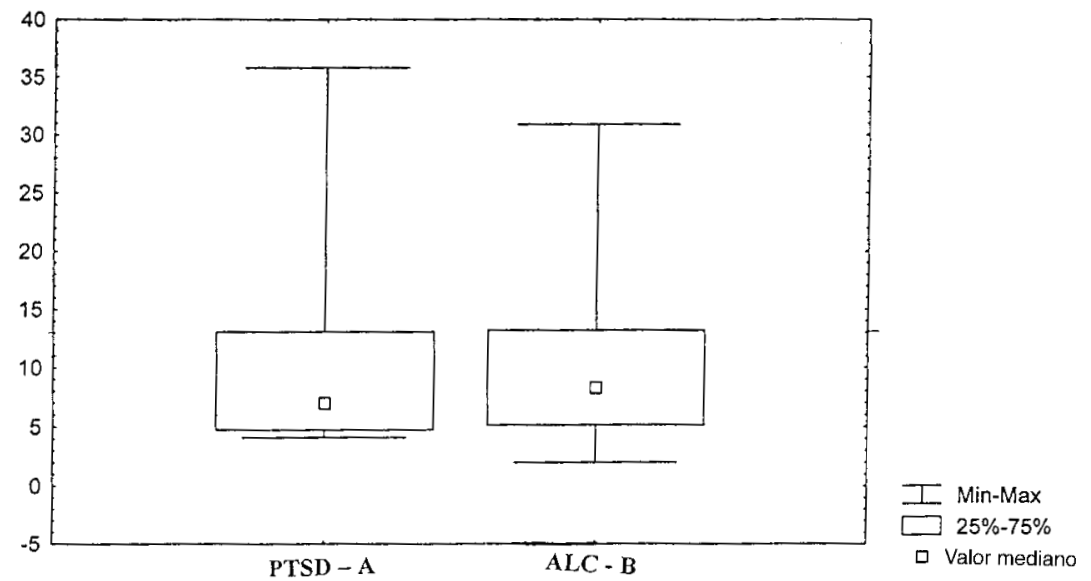

Figura 6. Niveles de ACTH en suero en el grupo de pacientes con PTSD (A) y en el grupo de pacientes con ambos diagnósticos, alcoholismo y PTSD (B). 


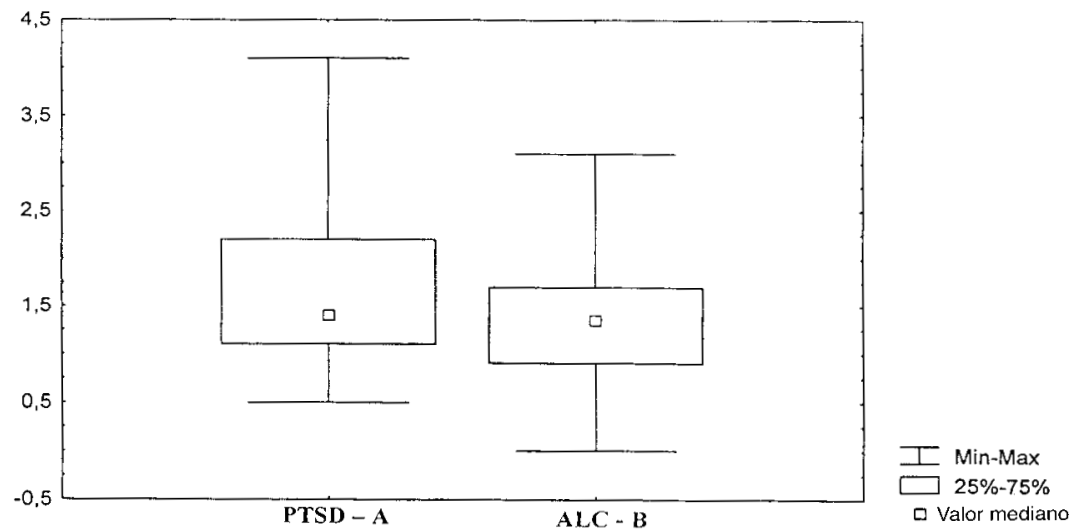

Figura 7. Niveles de TSH en suero en el grupo de pacientes con PTSD (A) y en el grupo de pacientes con ambos diagnósticos, alcoholismo y PTSD (B).

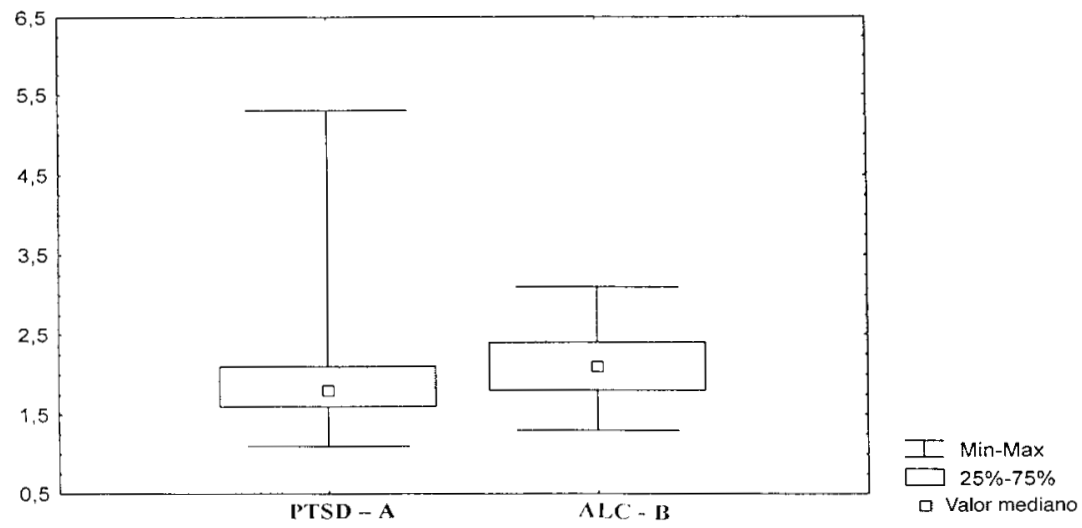

Figura 8. Niveles de T3 en suero en el grupo de pacientes con PTSD (A) y en el grupo de pacientes con ambos diagnósticos, alcoholismo y PTSD (B).

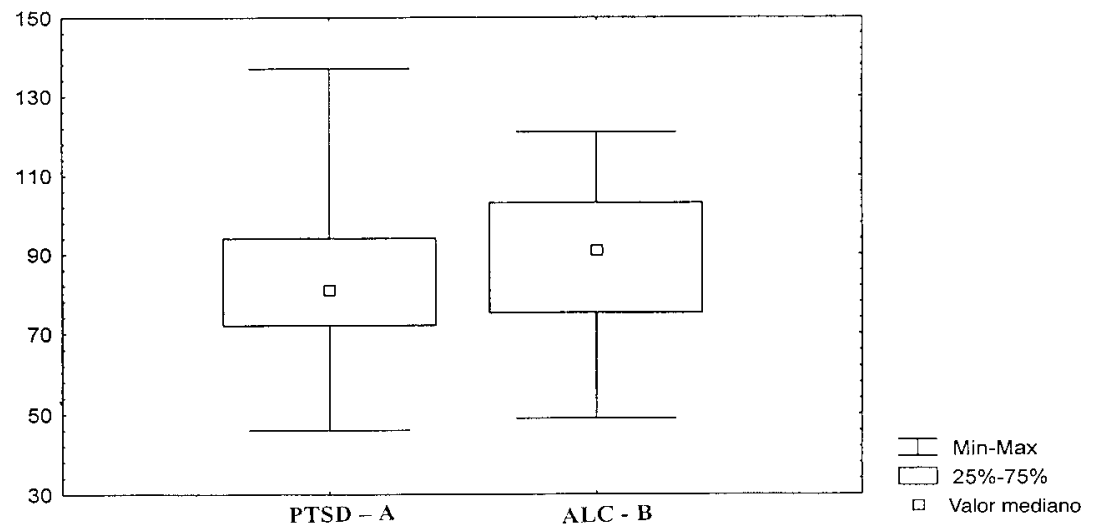

Figura 9. Niveles de T4 en suero en el grupo de pacientes con PTSD (A) y en el grupo de pacientes con ambos diagnósticos, alcoholismo y PTSD (B). 

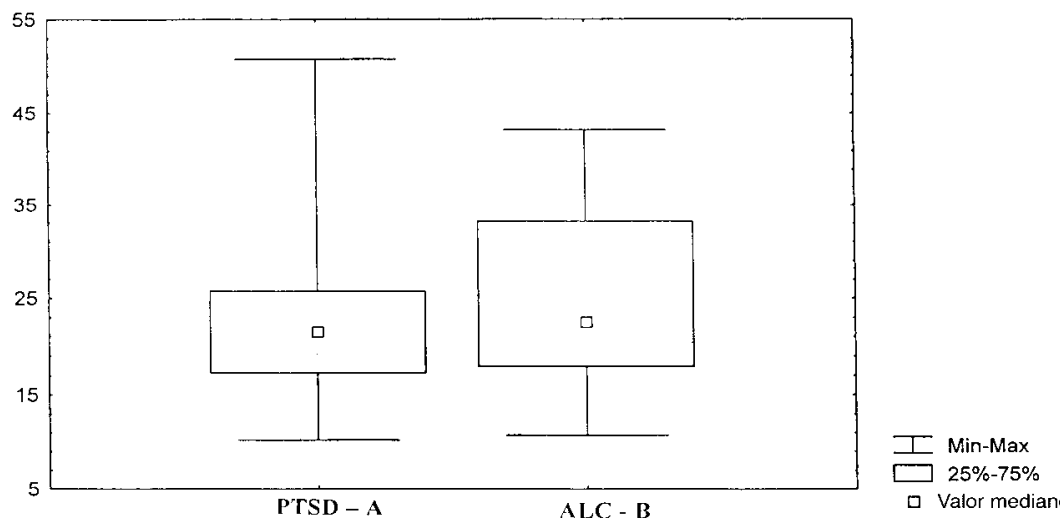

Figura 10. Niveles de testosterona en suero en el grupo de pacientes con PTSD (A) y en el grupo de pacientes con ambos diagnósticos, alcoholismo y PTSD (B).

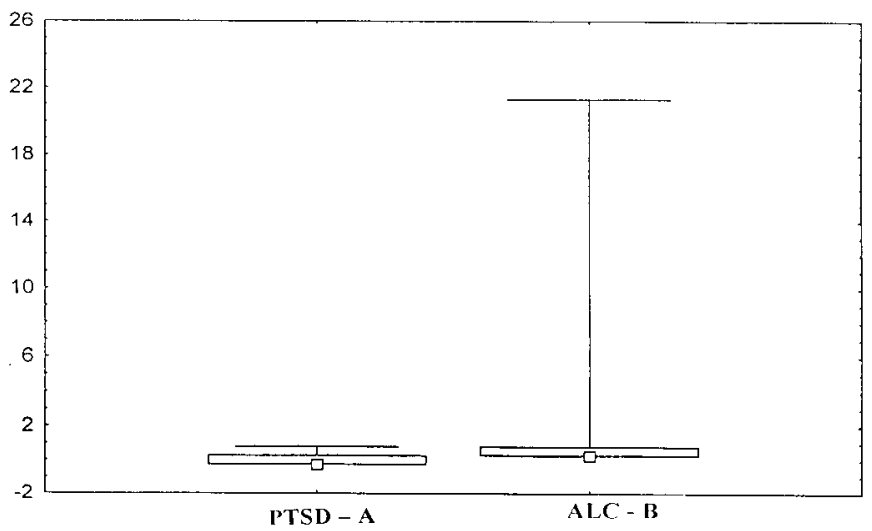

T Min-Max

ㅁ valor mediano

Figura 11. Niveles de hormona de crecimiento en suero en el grupo de pacientes con PTSD (A) y en el grupo de pacientes con ambos diagnósticos, alcoholismo y PTSD (B).

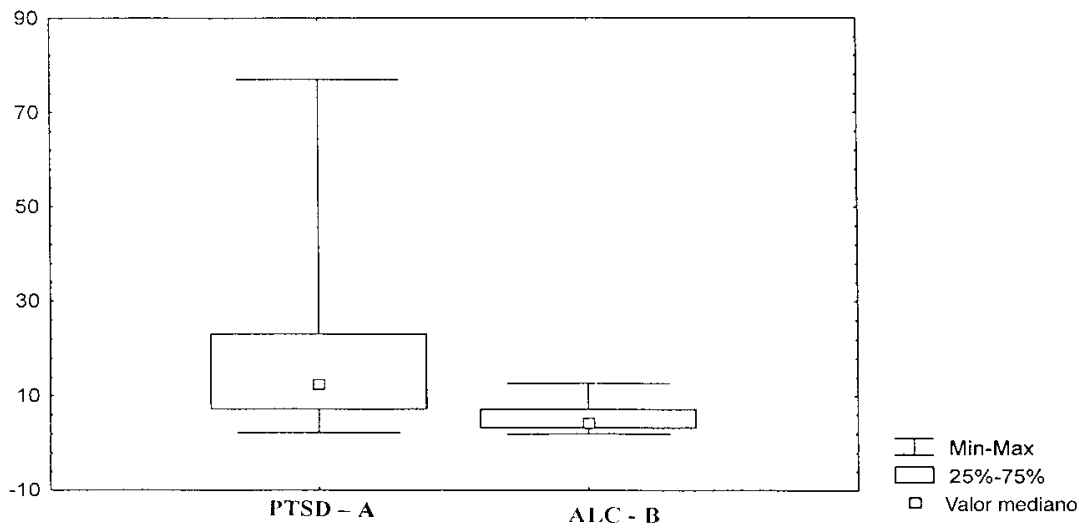

Figura 12. Niveles de prolactina en suero en el grupo de pacientes con PTSD (A) y en el grupo de pacientes con ambos diagnósticos, alcoholismo y PTSD (B). 


\section{Discusión}

Es bien sabido que los factores emocionales, físicos y químicos pueden originar una respuesta de estrés, con la activación del sistema endocrino, basado en la entrada desde el sistema límbico y el córtex al hipotálamo, teniendo como resultado la liberación de factores estimulantes o inhibidores que regulan la secreción de la glándula hipófisis y que como consecuencia moderan la actividad de otras glándulas endocrinas (suprarrenales, tiroides, gonadales). En pacientes que padecen PTSD, la función del eje HHA es especialmente importante, pues modera la secreción de hormonas glucocorticoides, que desempeñan un importante papel en el bloqueo del efecto de los agentes causantes del estrés que desencadena su liberación (Oitzl et al. 1997). No deberíamos pasar por alto los otros ejes, como el HHT (hipotálamo-hipófisis-tiroides) de gran importancia para las funciones mentales y el eje HHGn (hipotálamo-hipófisis-gonadal) que tiene un papel destacado en el desarrollo de los procesos mentales y en las pautas de conducta, tales como la conducta agresiva, que puede tener conexión con la estimulación androgénica. Tampoco deberíamos omitir el papel de la hormona del crecimiento, actuando en la dirección contraria a la de las hormonas glucocorticoides, que también pueden influir en la manifestación de conducta agresiva y en las alteraciones en el estado de ánimo. También es sabido que la hiperprolactinemia produce un aumento de la irritabilidad (Friedman 1991).

El Trastorno de Estrés Postraumático se define como un estado que puede darse en individuos que hayan estado expuestos a acontecimientos traumáticos extremos. El síntoma fundamental que domina el cuadro clínico es la ansiedad aguda, pero se dan también alteraciones en la capacidad de con- centración, pérdida de interés por la vida cotidiana, aturdimiento emocional, insomnio y alteraciones depresivas en el estado de ánimo (DSM-IV, American Psychiatric Association 1994). Las investigaciones sobre el sistema neuroendocrino en la depresión movieron a realizar investigaciones sobre el sistema neuroendocrino en el PTSD, especialmente en cuanto al papel del cortisol, si bien no debería ignorarse el papel de otros factores neuroendocrinos. Debemos tener en cuenta también la duración e intensidad del estrés, la exposición previa al estrés así como los factores sociales y genéticos. Los efectos de todos estos factores pueden alterar drásticamente la respuesta de los ejes neuroendocrinos al estrés, lo que da como resultado un incremento del riesgo de desarrollar PTSD tras la exposición al trauma.

Numerosas investigaciones en el campo del PTSD y el sistema neuroendocrino, en especial la respuesta del eje HHA al estrés, llevan a considerar que el tipo y la intensidad de los causantes del estrés son también de gran importancia y pueden ocasionar la disfunción del eje HHA. Se ha establecido que las alteraciones del eje HHA producen estados como depresión, alcoholismo, síndrome premenstrual, anorexia nerviosa y esclerosis múltiple (Yehuda 1991, Vrkljan et al. 1997). La respuesta anormal del cortisol a la activación del eje HHA se ha descrito tanto en el caso del estrés como en el de la depresión. En los casos de estrés crónico se presentan mayoritariamente niveles bajos de cortisol, aunque también se han descrito niveles elevados, así como la carencia de supresión de dexametasona (Yehuda et al. 1995, Owens et al. 1996, Lukas \& Mendelson 1988).

Los pacientes que padecen PTSD muestran también un incremento en los niveles en suero de ACTH. Se ha detectado una hipersecreción de ACTH en pacientes con depresión (Owens et al. 1996). El ritmo cir- 
cadiano normal de cortisol liberado se encuentra directamente influenciado por el ACTH, que se encuentra a su vez bajo la influencia de otros factores físicos y emocionales, que pueden llevar al incremento de los niveles en suero de ACTH. El mecanismo de respuesta negativa juega un importante papel en este sistema, pues al producir glucocorticoides y a través de los efectos que estos generan, influye en la producción de ACTH y CRF (Nash y Maickel 1988; Yehuda et al. 1995).

La ausencia de inhibición de la reacción negativa muestra que el PTSD potencia la estimulación crónica del CRF hipotalámico, que es respaldado por el incremento de secreción de la hipófisis. El modelo de disminución de la inhibición por medio de la reacción negativa concuerda con la observación clínica de que los pacientes que padecen de PTSD son especialmente reactivos al estrés y de que con frecuencia, muestran una respuesta biológica exagerada a acontecimientos no traumáticos (Mason et al. 1986).

El efecto directo del alcohol sobre el eje HHA conduce de forma gradual al desarrollo de alteraciones sobre el mismo, originando el conocido síndrome del "seudo-Cushing”, con un aumento del cortisol (Veldman \& Meinders 1996).

Los resultados de nuestro estudio muestran la producción de fuertes alteraciones circadianas del cortisol en ambos grupos de individuos objeto de estudio dándose una carencia en el test de supresión de dexametasona, así como un incremento en los niveles de cortisol y ACTH, lo que hace pensar en una disfunción del eje HHA.

Los resultados obtenidos muestran también que la afectación del eje HHA es más marcada en pacientes que padecen tanto de PTSD como de alcoholismo. Los niveles séricos de las hormonas TSH, T3 y T4 tam- bién llevan a considerar una disminución de la respuesta de TSH a TRH, que también es más acusada en pacientes con PTSD y alcoholismo. Los niveles séricos de hormona del crecimiento, testosterona y prolactina muestran también un incremento, lo que hace pensar igualmente en afectación de los ejes HHT y HHGn, proporcionándonos una mejor comprensión de la conducta en alto grado incontrolada de nuestros pacientes, especialmente en aquellos que padecen tanto de PTSD como de alcoholismo.

\section{Conclusión}

Podemos decir de forma concluyente que nuestras investigaciones confirman la tesis de afectaciones del el sistema neuroendocrino, con un desarrollo de perturbaciones en el mecanismo de la reacción negativa.

Podemos considerar al alcohol uno de los agentes causantes de estrés, al presentar un influencia significativa en la producción de cortisol, dando como resultado su hiperproducción y creando además perturbaciones en la producción de la hormona tiroidea. También es considerable su influencia en cuanto a la producción de testosterona y de hormona del crecimiento, cuyos niveles en suero, en ambos casos, presentan un incremento en los alcohólicos.

Esa es la razón por la que no podemos ignorar el hecho de que las hormonas procedentes del eje hipotálamo-hipófisis-suprarrenal se activan igualmente tanto durante la exposición al estrés como durante el consumo de alcohol, pero tambien el sistema opioide es activado provocando un aumento de las ansias por consumir alcohol bajo la influencia directa de los receptores dopaminérgicos activos que liberan dopamina, lo 
que aumentan los efectos del alcohol, creando un círculo vicioso que finalmente conduce a alteraciones en el eje HHA (Charness 1989, Jenison et al. 1992).

Por ello, podemos decir que los acontecimientos traumáticos y el alcohol, como agentes causantes de estrés, juntos potencian de forma directa la afectación de los ejes neuroendocrinos, agravando de forma significativa la influencia terapéutica directa y el proceso de tratamiento.

\section{Bibliografía}

American Psychiatric Association: Diagnostic and Statistical Manual of Mental Disorders, IV. American Psychiatric Association, Washington, 1994.

BONNEAN, R.H., KIECOLT-GLASER, J.K., GLASER, R. Stress-induced modulation of the immune response. Ann Ny Acad. Sci., 594: 253-269, 1990.

CHARNESS, M.E. Ethanol and opoid receptor signaling. Experientia 45: 418-428, 1989.

CONSTANTI, A., BARTKE, A., KHARDORI, R. Basic Endocrinoloy for students of Pharmacy and Allied Clinical Health Sciences. Amsterdam: Harwood Academic Publishers, 1998.

DAVIDSON, R.T., KUDLER, H.S., SAUNDERS, W.B. et al. Symptom and Comorbidity patterns in World War II and Vietnam veterans with posttraumatic stress disorder. Comprehensive Psychiatry 31: 162-170, 1990.

DE KLOET, E.R., RATKA, A., REUL, J.M.H.M., SUTANTO, W. Corticosteroid receptor typs in brain, regulation and putative function. In: Ganong, W.P., Dalman, M.F., Roberts, J.L. (eds.) The HPA axis revisited. Ann NY Acad. Sci., 31: 512-522, 1987.

EMANUELE, N., EMANUELE, M.A. The endocrine system: Alcohol alters critical hormonal balance. Alcohol Health \& Research World, 21 (1): 53-64, 1997.

FRIEDMAN, M.J. Biological approaches to the diagnosis and treatment of posttraumatic stress disorder. Journal of Traumatic Stress, 4: 67-91; 1991.

GOLD, P.W., LORIAX, D.L., ROY, A. et al. Responses to corticotropinreleasing hormone in the hypercortisolum of depression and Cushing's disease, New Eng. J. Med., 314: 1329-1334, 1986.

JENISON, K.M. The impact of stressful life events and social support on drinking among older adults: A general population survey. International Journal of Aging and Human Development 35(2): 99-123, 1992.

KHANSARI, D.N., ANTHONY, J.M., ROBERT, E.F. Effects of Stress on the immune sistem. Immuno Today 1990, 11: 170-175.

LOOSEN, P.T., Thyroid function in affective disorders and alcoholism. Endocrinol Metab. Clin. North AM 17(1): 55-82, 1988.

LUKAS, S.E., MENDELSON, J.H. Electroencephalographic activity and plasma ACTH during ethanol-induced euphoria. Biol. Psych. 23(2): 141-148, 1988.

MASON, J.W., GILLER, E.L., KOSTEN, T.R., OSTROFF, R.B., PODDL, L. Urinary-free Cortisol levels in posttraumatic stress disorders patients. J. Nerv. Ment. Dis. 174: 159, 1986.

NASH, J.F., MAICKEL, R.P. The role of Hypothalamic -pituitary- adrenocortical axis in post stress induced ethanol consummation by rats. Progress in Neuro-psychopharmacology and Biological Psychiatry 12: 653-671, 1988

OITZL, M.S., VAN HAARST, A.D., DE KLOET, E.R. Behavioral and neuroendocrine responses controlled by the concerted action of central mineralocorticoid (MRs) and glucorticoid (GRs) receptors. Psychoneuroendocrinology, 22 (Suppl): 87-93, 1997.

OWENS, M.J., PLOTSKY, P.M., NEMEROFF, C.B. CRF, the HPA axis and depression: role of early life events. $27^{\text {th }}$ ISPNE Congress Casais, Portugal, 1996.

SADAWA. S.W., PAK, A.W. Stress related problem drinking and alcohol problems: A longitudinal study and extension of Marlatt's model. Canadian Journal of Behavioral science 25(3): 446-464, 1993.0

SOUTHWICK, S.M., KRYSTAL, J.H., JOHNSON, D.R., CHARNEY, D.S., Neurobiology of posttraumatic stress disorder. Review of Psychiatry, vol. 11, A. Tasman, M.B. Riba, (eds.) p. 347. American Psychiatric Press, Washington, 1992. 
TSIGOS, C., CHROUSOS, G.P. The neuroendocrinology of the stress response. W. Hunt, S. Zakharis (eds). Stress, Gender and Alcohol-seeking Behavior. National Institute on Alcohol Abuse and Alcoholism Research Monograph $n .^{\circ}$ 29. Bethesda MD: the institute, 1995.

VELDMAN, R.G., MEINDERS, A.E. On the mechanism of alcohol-induced pseudo-Cushing's Syndrome. Endocrine Reviews, 17: 262-268, 1996.

VRKLJAN, M., THALLER, V., STANCIC, V., TOMAC, A., KUSIĆ, Z. Plasma and urinary cortisol level in patients with posttraumatic stress disdorder (PTSD) and major depressive disorder (MDD) Psychoneuroendocrinoloy 22 (Supll 2): 5213, 1997.

WAND, G.S., DOBS, A.S. Alterations in the hipothalamic-pituitary-adrenal axis in actively drinking alcoholics. J. Clin. Endocrinol Metab. 72(6): 1290-1295, 1991.

YEHUDA, R. Hypothalamic-pituitary-adrenal axis alteration in posttraumatic stress disorder: an alternative model of stress. Proceedings of $25^{\text {th }}$ ISPNE Congress, August 18-22, 1996 Cascais, Portugal, 1996: 99.
YEHUDA, R., GILLER, E.L., SUTHWICK, S.M, LOWY, M.T., MASON, J.W. Hypothalamic-pituitary-adrenal dysfunction in posttraumatic stress disorder. Biol. Psychiatry 30: 1031-1048, 1991.

YEHUDA, R., BOISOENAU, D., LOWY, M.T., GILLER, E.L. Jr. Dose response changes in cortisol and lymphocyte glucocorticoid receptors following dexamethasone administration in combat veterans with and without posttraumatic stress disorder. Arch. Gen. Psychiatry, 52: 283-293, 1995.

Dirección para correspondencia:

Vlatko Thaller, Prof. Dr. SC.

University Department of Psychiatry

KB "Sestre Milosrdnice"

Referal Centre for Alcoholism

Ministry of Health

HR-10000 Zagreb, Vinogradska c. 29

E-mail: vlatko.thaller@zg.tel.hr CROACIA 